\title{
Micromechanical behaviors related to confined deformation in pure titanium
}

\author{
Runguang $\mathrm{Li}^{1}$, Youkang Wang ${ }^{1}$, Xiaojing Liu ${ }^{1}$, Shilei Li ${ }^{1}$, Qing $\operatorname{Tan}^{1}$, Wenjun Liu ${ }^{2}$, Xing Fang ${ }^{3}$, Yan-Dong \\ Wang 1 , * \\ ${ }^{1}$ Beijing Advanced Innovation Center for Materials Genome Engineering, State Key Laboratory for Advanced \\ Metals and Materials, University of Science and Technology Beijing, Beijing, 100083, China \\ ${ }^{2}$ X-ray Science Division, Advanced Photon Source, Argonne National Laboratory, Argonne, IL 60439, USA \\ ${ }^{3}$ State Key Laboratory of Heavy Oil Processing, China University of Petroleum, Beijing, 102249, China \\ * Corresponding author. \\ E-mail addresses: ydwang@ustb.edu.cn (Y.-D. Wang).
}

Keywords: synchrotron, pure titanium, laser shot peening, twinning

\begin{abstract}
Confined deformation, e.g. mechanical twinning, shear banding, and Lüders banding, etc. was extensively observed in metals and alloys with low stacking-fault energies, especially under complex loadings, governing the mechanical properties. It is often accompanied with gradient microstructures to accommodate the stress concentrations. Understanding the micromechanical behaviors of structural materials having confined deformation is important for evaluating the structural stabilities of engineering components. Synchrotron-based techniques provide powerful tools for multiscale microstructural characterization owing to their good resolution in real/reciprocal space, fast data collection/processing and flexible application scenarios. In this paper, the synchrotron-based high-energy X-ray diffraction (HE-XRD) and microdiffraction ( $\mu \mathrm{XRD}$ ) techniques in combination with traditional characterization methods are used to reveal the deformational gradient structures/stresses under different loading modes in multiscale. The structure/stress gradients induced by laser shot peening treatment and the deformation twins generated during uniaxial tensile loading in pure titanium were systematically studied by HE-XRD and $\mu \mathrm{XRD}$, in order to elucidate the accommodating role of the deformational structures subjected to various confined scenarios. The new finding regarding the
\end{abstract}


micromechanical behaviors related to confined deformation contributes to the in-depth understanding of related complex deformation behaviors.

\section{Introduction}

The strain localization in metallic materials is mainly governed by both confined deformation geometry and crystallographic features [1]. The formation of strain localization is preceded by the creation of obstacles to homogeneous deformation, which physically constitutes a type of instability in ductile materials. The plastic deformation heterogeneity is enhanced by the complex interactions of dislocation slip and mechanical twinning, generating the local hardening/softening due to crystal anisotropy and change in deformation path, especially in low stacking-fault energy metals and alloys. These samples undergoing a dense shear strain may easily produce banding structures (e.g. shear bands) with a jump in both grain orientation and stress, having the specific substructure patterns clearly distinguished from the surrounding matrix. The further deformation is constrained by the above-mentioned banding structures, causing alteration of micromechanical behavior, which is here referred as the confined deformation.

The conventional characterization methods, e.g. the transmission electron microscopy (TEM) and the electron back-scattered diffraction (EBSD) or electron channeling contrast (ECC) technique in scanning electron microscopy (SEM) etc., were widely employed in the ex situ study of deformation microstructures. The origin of mechanical heterogeneity as a complex irreversible change in substructures under various loading conditions is usually involved with the formation of various zones with localized strains, e.g. twinning, shear banding, and Lüders banding, etc. Stress concentration was often observed near their boundaries. The mechanism on the formation and evolution of the above substructures is complicated as local hardening/softening usually occurs. Meanwhile, the micron-scale inhomogeneous stress field significantly influences the microstructure under further loading.

In recent decades, the laser shot peening (LSP) technique was developed and attracted great attentions since it introduces significant compressive stress distributing deeply inside a metal or alloy and thus greatly increases the fatigue life of components. Essentially, it is a cold working process where pulses hit the surface with high power intensity and generate shock waves [2]. These waves make the surface plastically deformed and extend the compressive stresses underneath far from the subsurface [3], resulting in a strain rate exceeding $10^{6} \mathrm{~s}^{-1}[4,5]$. Based on extensive industry experiences, LSP can effectively delay the initiation and propagation of fatigue cracks by introducing compressive residual stresses to a depth of millimeters $[2,6]$. The fatigue cracks tend to initiate at surface or near surface where are subjected to tensile stress [7] and propagate toward the material interior. The LSP technique is now widely used in engineering components, such as the turbine discs and blades in aircraft engines, to improve their fatigue performance [8-10]. Physically, various gradients in both 
microstructures, e.g. twins, shear bands, etc., and stress were introduced into the surface and near-surface layers via peening treatment, which change the properties of components in the following confined deformation. This process is complex and still not well revealed due to the limitation of spatial resolution in both reciprocal and real sample spaces using the above mentioned conventional characterization methods.

The characterization of the mesoscopic/microsopic deformation structures and stresses is necessary for understanding the defect patterns and intrinsic mechanisms of metals and alloys from a more comprehensive view. Advanced characterization techniques, such as synchrotron-based techniques, provide powerful tools for the in situ exploration of micromechanical behavior under various loading conditions and temperature environments [11-14], playing a significant role in material researches nowadays. Here the synchrotron based HE-XRD and $\mu$ XRD were employed to study the structure/stress gradients induced by LSP and deformation twinning in pure titanium, in order to demonstrate the capacity and efficiency of the synchrotron-based techniques in the studying of scientific issues related to plastic strain heterogeneity.

\section{Experiments}

\section{Laser shot peening}

The $99.995 \%$ purity titanium plates used in this study is in single phase (a-Ti) with close-packed hexagonal (HCP) structure and an average grain size of $30 \mathrm{~mm}$. The as-received pure titanium sample for LSP is a plate with a thickness of $8 \mathrm{~mm}$. LSP treatment was conducted on both sides of the fully annealed and stress-free plate to generate symmetric deformation gradients. Fig. 1a shows the schematic of the LSP technique. Laser pulse passes through the transparent confining layer and is absorbed by the absorbent layer. The absorbent material immediately vaporizes and forms plasma with high temperature and high pressure moving towards the confined layer. The rapid expanded plasma is trapped by the confined layer and generates a backward shock wave against the work piece. In the study, the pulsed laser was produced by a Nd: YAG laser with a wavelength of $1064 \mathrm{~nm}$ and a pulse duration of $20 \mathrm{~ns}$. The laser spot was $2.5 \mathrm{~mm}$ in diameter with a power density of $7 \mathrm{GW} / \mathrm{cm}^{2}$. The confined layer and absorbent layer was floating water (1 2 $\mathrm{mm}$ in thickness) and Al coating (0.1 $\mathrm{mm}$ in thickness), respectively. The ratio of overlapping laser pulses is $50 \%$.

\section{$\underline{\text { HE-XRD experimental }}$}




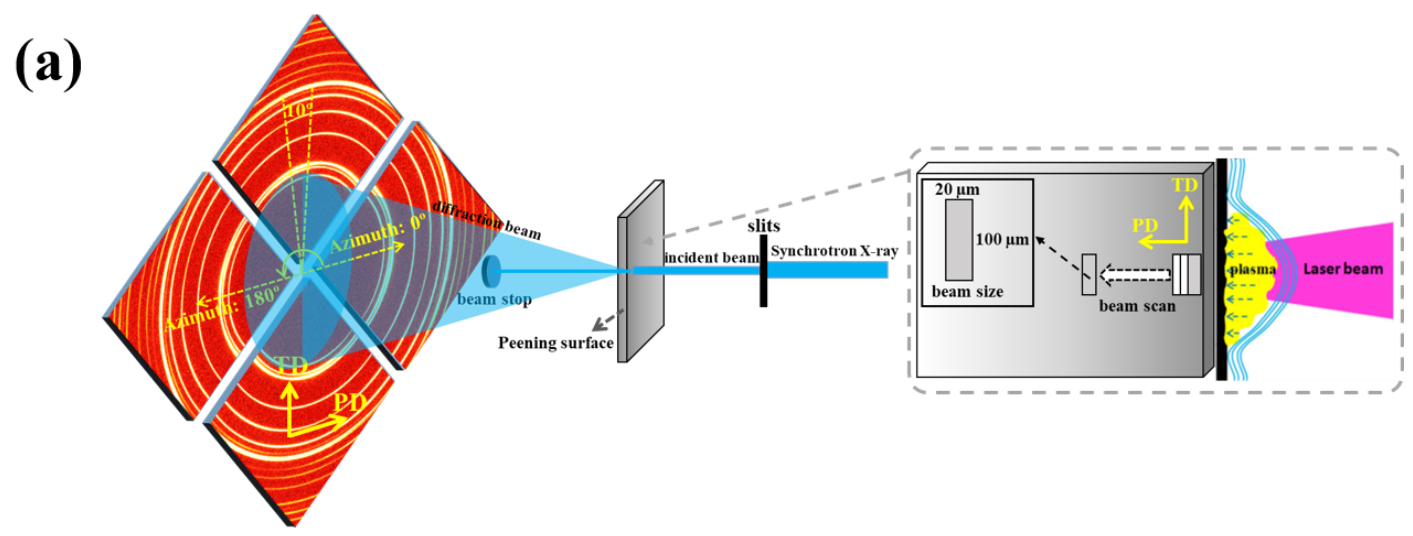

(b)
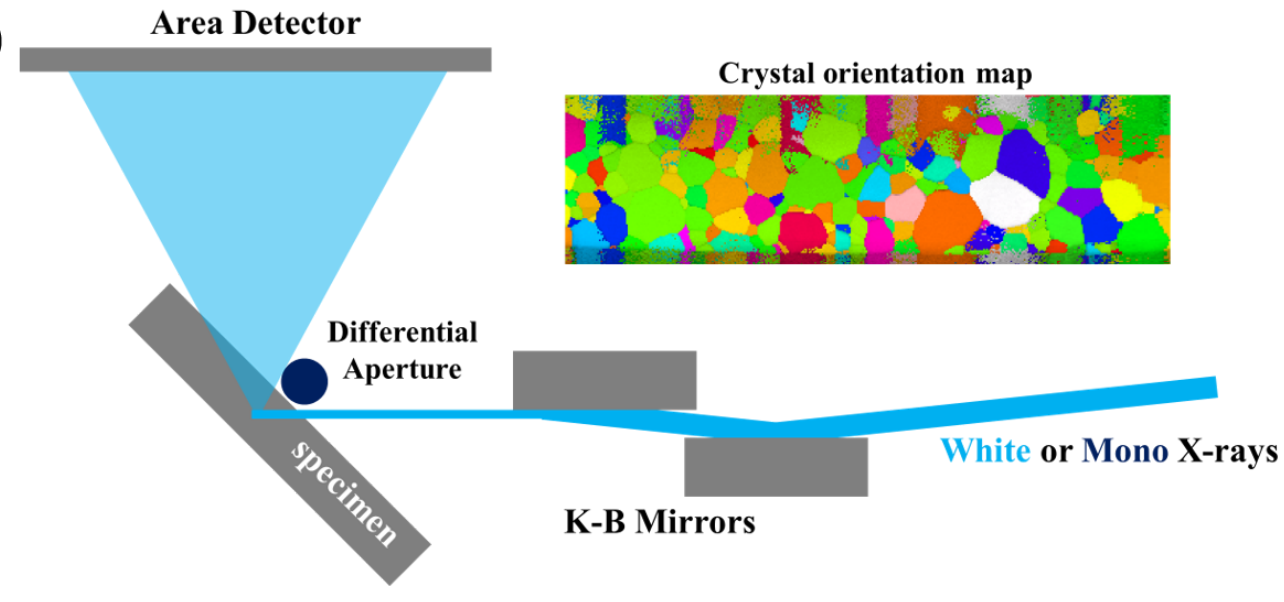

K-B Mirrors

Figure 1. Schematics of synchrotron-based techniques. (a) HE-XRD for characterizing the peening induced gradients.

(b) 3D $\mu$ XRD for studying the local strain gradients of deformation twins.

The HE-XRD experiments for the peened titanium were conducted on the beamline 1-ID-E of the Advanced Photon Source, Argonne National Laboratory (APS, ANL) with the experimental schematic shown in Figure 1a. Piece-shape sample was machined along the peening direction (PD) from the as received peened plate. The sample was mounted with the PD perpendicular to the X-ray incident direction. Diffraction patterns were recorded by four two-dimensional (2-D) detectors installed in front of the sample with $\sim 2.5 \mathrm{~m}$ away. The X-ray wavelength used in this study was $0.1730 \AA$. For a high spatial resolution of gradient in PD, slits were used to restrict the beam size to $20 \mathrm{~mm} \times 100 \mathrm{~mm}$ with the beam scanning along PD by moving the sample frame left stepwise with the translation sample stage, thus the incident X-ray beam can cover different locations along sample gage spot by spot with a displacement step of $20 \mathrm{~mm}$ along the PD to cover the entire gradients. The 
lattice parameters for pure titanium are: $\langle\mathrm{a}\rangle=2.951 \AA,\langle\mathrm{c}\rangle=4.687 \AA$, and c/a ratio of 1.587. All experiments were conducted at room temperature. The data from the section with the scattering vector parallel to transverse direction (TD) and PD were integrated for calculating the lattice strains. The lattice strain $\varepsilon$ of plane ( $h k i l)$ was determined by the relative change in the corresponding lattice plane spacing, $\varepsilon_{h k i l}=d(h k i l) / d_{0}(h k i l)-1$, where $d(h k i l)$ and $d_{0}(h k i l)$ are the interplanar spacing of the $\{h k i l\}$ plane before and after an applied strain. The Bragg angle $\theta_{\text {hkil }}$ peak width, represented by the full width at half maxima (FWHM) was used for quantifying strain heterogeneity, which was obtained through correcting the instrumental broadening with Ceria powders (Voigt function is used for peak fitting).

\section{$\underline{3 \mathrm{D} \text { synchrotron } \mathrm{X} \text {-ray microdiffraction }(3 \mathrm{D} \mu \mathrm{XRD})}$}

The in situ 3D $\mu \mathrm{XRD}$ experiments on deformation twinning of pure titanium were performed at the beamline 34ID-E of APS, ANL. As the experimental schematic shown in Figure 1b, spatially resolved diffraction measurements were conducted by using differential aperture X-ray microscopy with a polychromatic or monochromatic X-ray microbeam being focused by a pair of the Kirkpatrick-Baez mirrors to a small spot of 0.5 $\mu \mathrm{m} \times 0.5 \mu \mathrm{m}$, where the Laue diffraction patterns were recorded on a PerkinElmer area detector. The depth resolution was provided by a $100-\mu \mathrm{m}$-diameter platinum wire as a diffracted beam profiler (differential aperture) scanning along the incident beam on the specimen surface to analysis and finally obtain a crystal orientation map in the polychromatic mode with an accuracy of $0.01^{\circ}$, while the absolute lattice parameters and hence local elastic lattice strains inside matrix-grain/twin were quantitatively measured in the monochromatic energyscanning mode with an accuracy of $10^{-4}[15,16]$. Hence a high spatial resolution of measurement is achieved at the submicron level in all three dimensions, revealing the local strain gradients accommodating twinning deformation.

\section{Results and discussions}

\section{$\underline{\text { Stress/microstructure gradients induced by laser shot peening }}$}

Diffraction method is effective in the nondestructive characterization of internal stresses especially for pure metals excluding the influence of heterogeneities in composition. For LSP processed components, the actual depths of the peening-induced structure/stress gradients vary depending on the type and intensity of the processing conditions and the material properties.

Figure 2 displays the EBSD characterization results of the sub-surface layer before and after the LSP treatment. As the SEM images shown in Figure 2a and 2b, extensive primary and secondary twinning occurred under the severe peening surface layer during the LSP treatment, forming shear bands patterns crossing multi 
grains into the deep direction. The twins belong to compression type mostly and these deformation structures generate large intergranular/back stresses among different orientation grains and result in the strain heterogeneity in the deeper layers after the peening waves. Obvious misorientations were observed among the deformed grains as indicated in Figure 2e and 2f, which is consistent with the patterns of the deformation twin clusters.

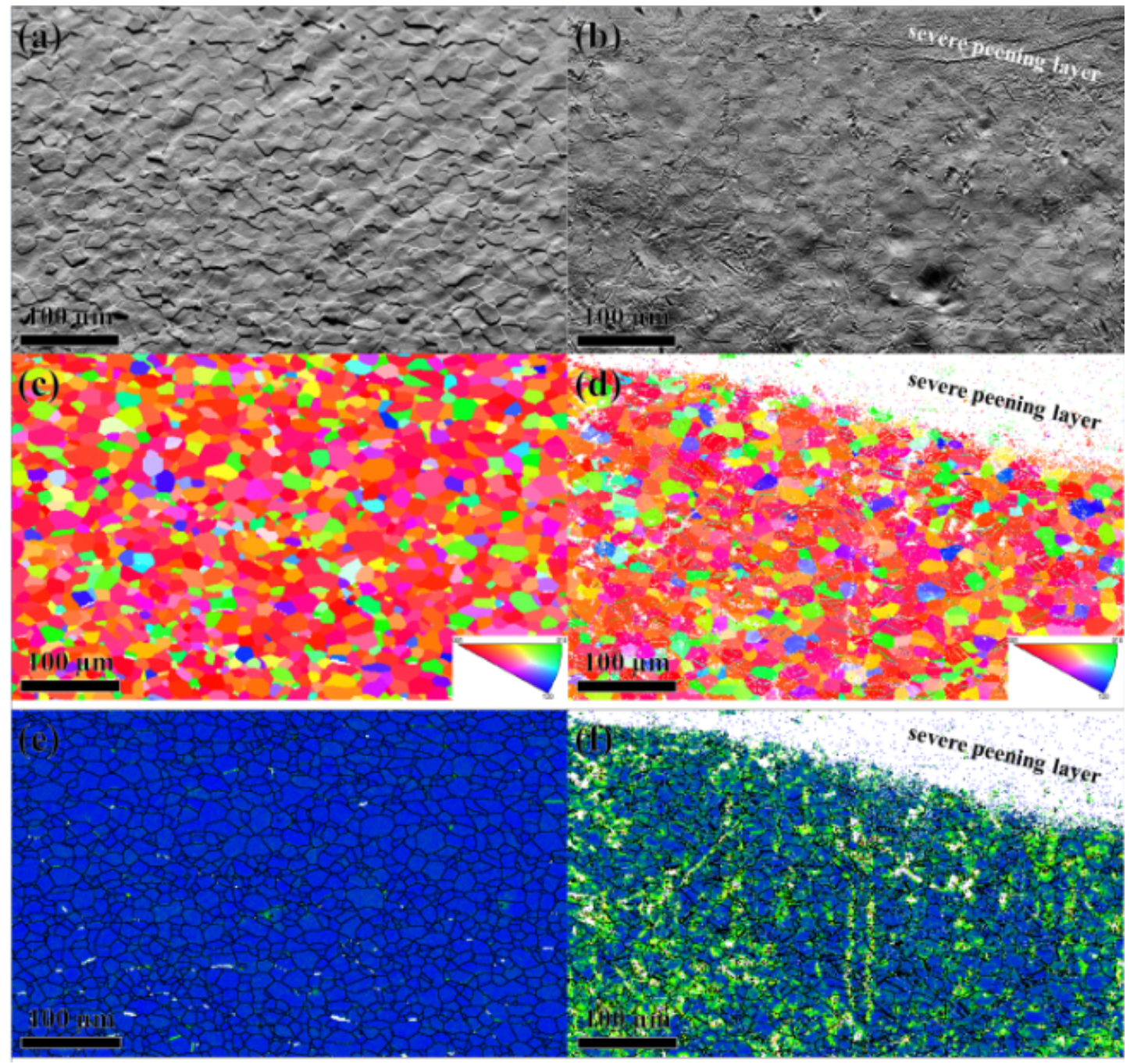

Figure 2. EBSD characterization results of the deformation microstructure before and after the LSP treatment in the sub-surface layer. (a, b) SEM images. (c, d) Orientation maps. (e, f) Local misorientation maps. 
The peening-induced internal strains were revealed by conducting the synchrotron based X-ray line-scan along PD. The lattice strains along TD and PD were calculated by integrating the TD/PD-azimuth sectors with an angle range of 10 degrees on Debye rings of the low indexed crystal planes, i.e. $\{10-10\},\{0002\},\{10-11\}$, and $\{10-12\}$. As plotted in Figure 3a, compressive TD lattice strains generated by peening induced deformation were observed with a depth of more than $2,500 \mathrm{~mm}$. The largest compressive lattice strain was in the surface layer. This is consistent with the previous report that the dynamic compressive stresses were highest on the surface and decrease with depth into the peening-material [4]. While interestingly, a sharp inverse "hook" followed by a broad "hook" was observed in the layer of about $60 \mathrm{~mm}$ in depth on the TD lattice strain profile, indicating the layer directly undergoing severe plastic deformations. The deeper broad "hook" is similar to the "hook" extensively observed on the residual stress profile of classic mechanical shot peening processed components $[17,18]$. It should be noted that the sharp inverse "hook" generated by LSP as shown in the compressive TD lattice strain profile just below the surface suggests a confined narrow layer with rapid stress change, which is difficult to be observed by conventional characterization methods without enough resolution in both spatial and stress measurement. The broad "hook" is considered to be due to the highly cold-worked material near the surface that fades quickly with increasing depth. The severe cold-work usually leads to instability of residual stresses, while it is not a concern for LSP as the hardened parts were arranged in a reasonable order, achieving a stability in its entirety. Actually, it creates the highest residual stress at the surface that rapidly decreases with the depth in the peening-deformed layer, followed by the broad "hook" and gradually decreases with distance below the "hook" cusp.
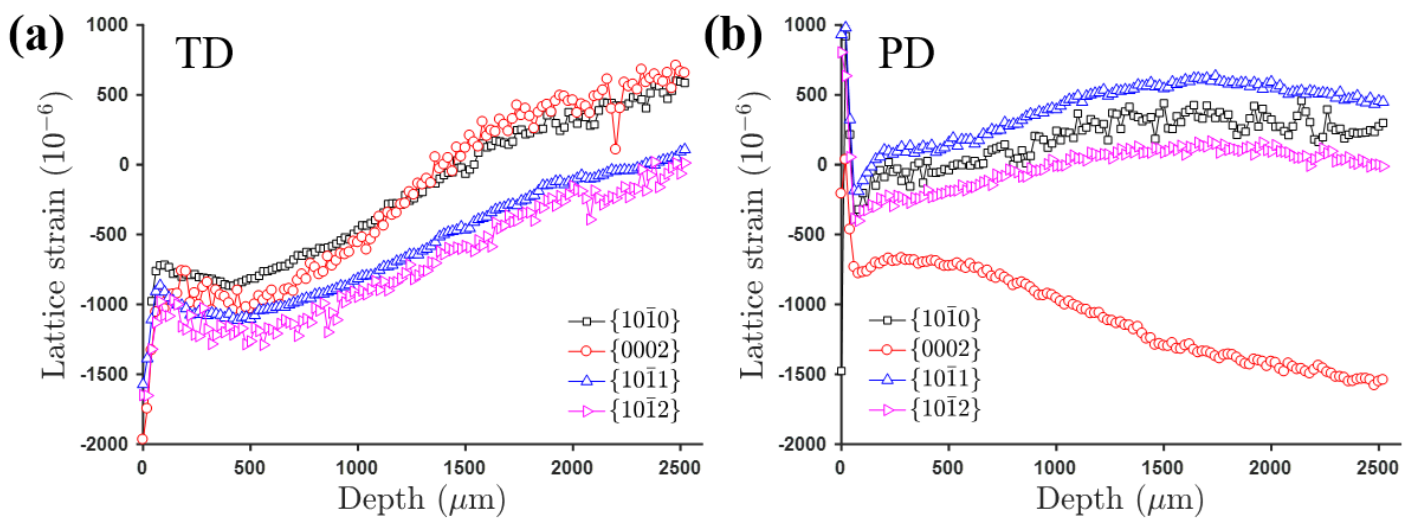

Figure3. Residual strain along TD (b) and PD (b) as a function of depth for the peening-titanium. 
The peening-induced PD stress as plotted in Figure $3 \mathrm{~b}$ is often ignored by the previous researches as the stress on the normal direction was usually regarded as inessential for the improvement of fatigue property. Using HE$\mathrm{XRD}$ technique, the internal strains along the PD were revealed in detail as well and surprisingly, the grains with [0002] axis nearly parallel to PD ([0002]/PD) in the deep layers was proved to undertake significant larger compressive lattice strains than other oriented grains in PD. While in the peening-deformed surface layers, the lattice strains of different orientation grains are in a highly consistence along both TD and PD. Detailed analyses on the twinning related orientation-dependent stress (especially on the [0002]//PD grains) in HCP materials will be reported elsewhere.

\section{Internal strain gradients in deformed titanium}

Twinning is the major deformation supplementary for the hexagonal-close-packed (HCP) structured metals $[19,20]$, accommodating localized strains where slipping is inhabited. Abrupt shearing always occurs with an approximate constant deformation tensor and sustains the deformability of components, resulting in the local incompatibility. Twin boundaries (TBs) play a crucial role accommodating local incompatibility in twinning deformation. TB migration was well established to be accomplished via the glide of Shockley partial dislocations on the twinning plane. Many researches have revealed that the TB in the HCP metals is consist of coherent TB and semi-coherent connections, i.e. basal/prismatic $(\mathrm{B} / \mathrm{P})$ interfaces $[21,22]$, which play a geometric role to reduce the strain heterogeneity of the boundaries.

The twinning deformation in pure titanium was studied by in situ synchrotron based $\mu \mathrm{XRD}$ technique. Crystal orientation maps of the dog-bone sample before (Fig. 4a) and after $2.4 \%$ tensile strain (Fig. 4b) were obtained via scanning a bulk interface by $\mu \mathrm{XRD}$ under polychromatic-mode. As the $\{0002\}$ pole figure colored corresponding to the orientation map of Fig. $4 \mathrm{~b}$ shows in Fig. 4c, the c axis of observed twins labeled as "TW1" and "TW2" is measured to be $\sim 84.9^{\circ}$ from the matrix grains G1 and G2, close to the theoretical calculation of $85.033^{\circ}$ and the twin boundaries are $\sim 2^{\circ}$ from the ideal $\{10-12\}$ coherent twin boundaries, which belong to the $\{10-12\}<\{10-11\}>$ type. There is a band region labeled as "TW3" in Fig. 4b whose Laue pattern is too noising to be detected. It is probably due to some nano-size micro twins as observed in TEM images as shown in Fig. 6 . The diffraction patterns of nano-size domains are hard to be derived from the $3 \mathrm{D} \mu \mathrm{XRD}$ datasets. 

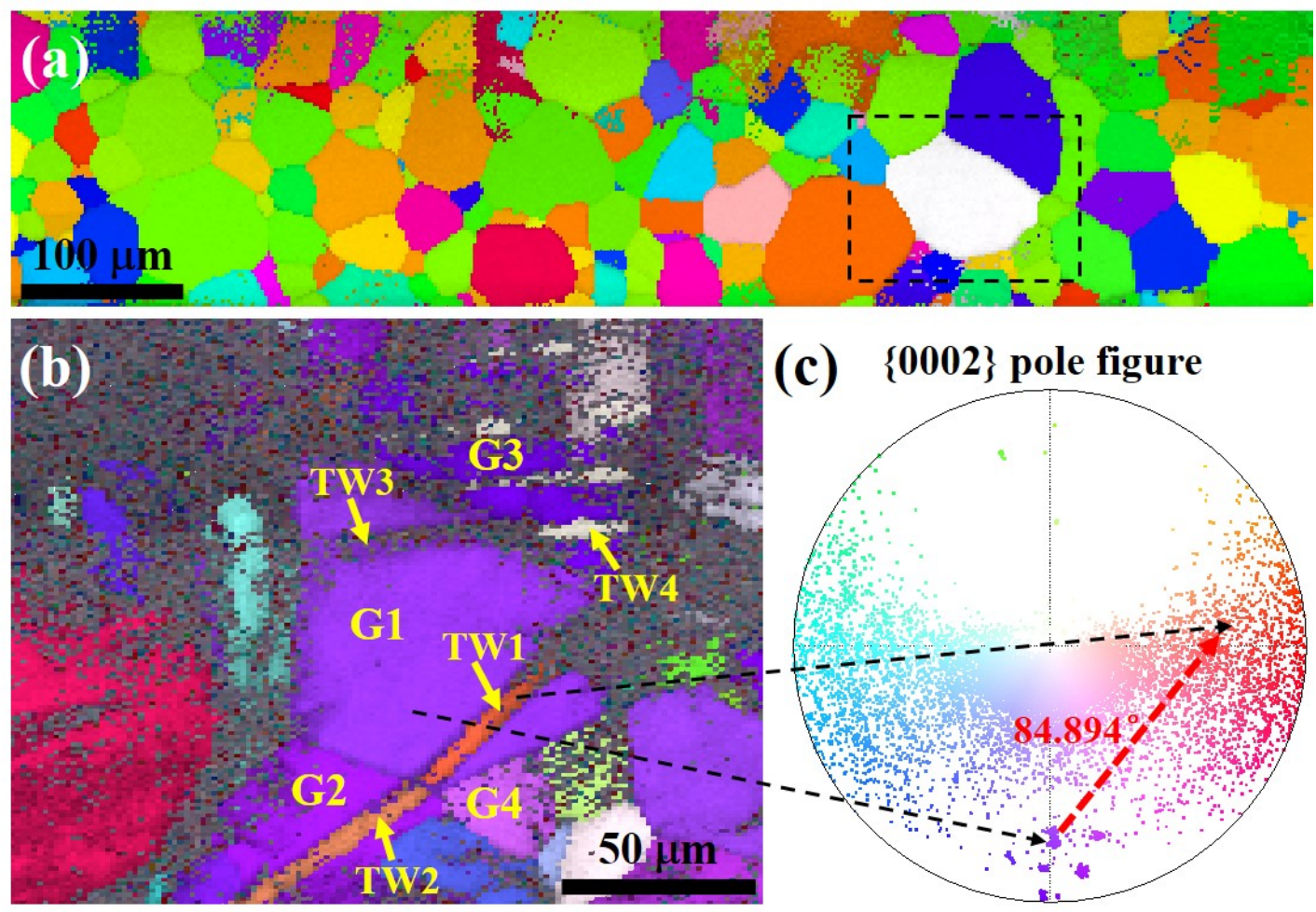

Figure 4. Twins and matrix grains characterized by uXRD under polychromatic-mode. Polycrystalline morphology of original (a) and deformed (b) pure titanium sample. (d) The $\{0002\}$ pole figure with same color map of (b).

The local stress state is of great value for understanding the physical mechanism of deformation. Figure 5 shows the lattice strain distribution of twins (TW1, TW2) and the matrix grains (G1, G2) measured using the monochromatic-mode of $\mu \mathrm{XRD}$. The measured lattice strain can be regarded as a combination of Poisson effect and internal back stress as the diffraction vector is nearly perpendicular to the sample surface. $\{6-5-1-4\}$ plane and $\{6-1-5-4\}$ plane are selected to characterize the internal strain distribution in TW1 and G1 and the results are plotted in Figure 5b-f. A much larger lattice distortion gradient in TW1 than in its parent grain G1, which is in agree with the work on MZ31 alloy by L. Balogh [23]. Stress relaxation was observed in the upper region of G1 near TW3, suggesting the accommodation role of nano-size localized strains near the grain boundary of HCP metal.

It needs to be emphasized that measuring the strain condition of subsurface grains in 3D is a particular and valuable capability of $\mu \mathrm{XRD}$ technique, enabling the material investigation in real bulk condition. This capability is important and urgent needed in the measurement of real local strain, which is strongly influenced by the surrounding medium and usually changes significantly when exposed in free surface. 

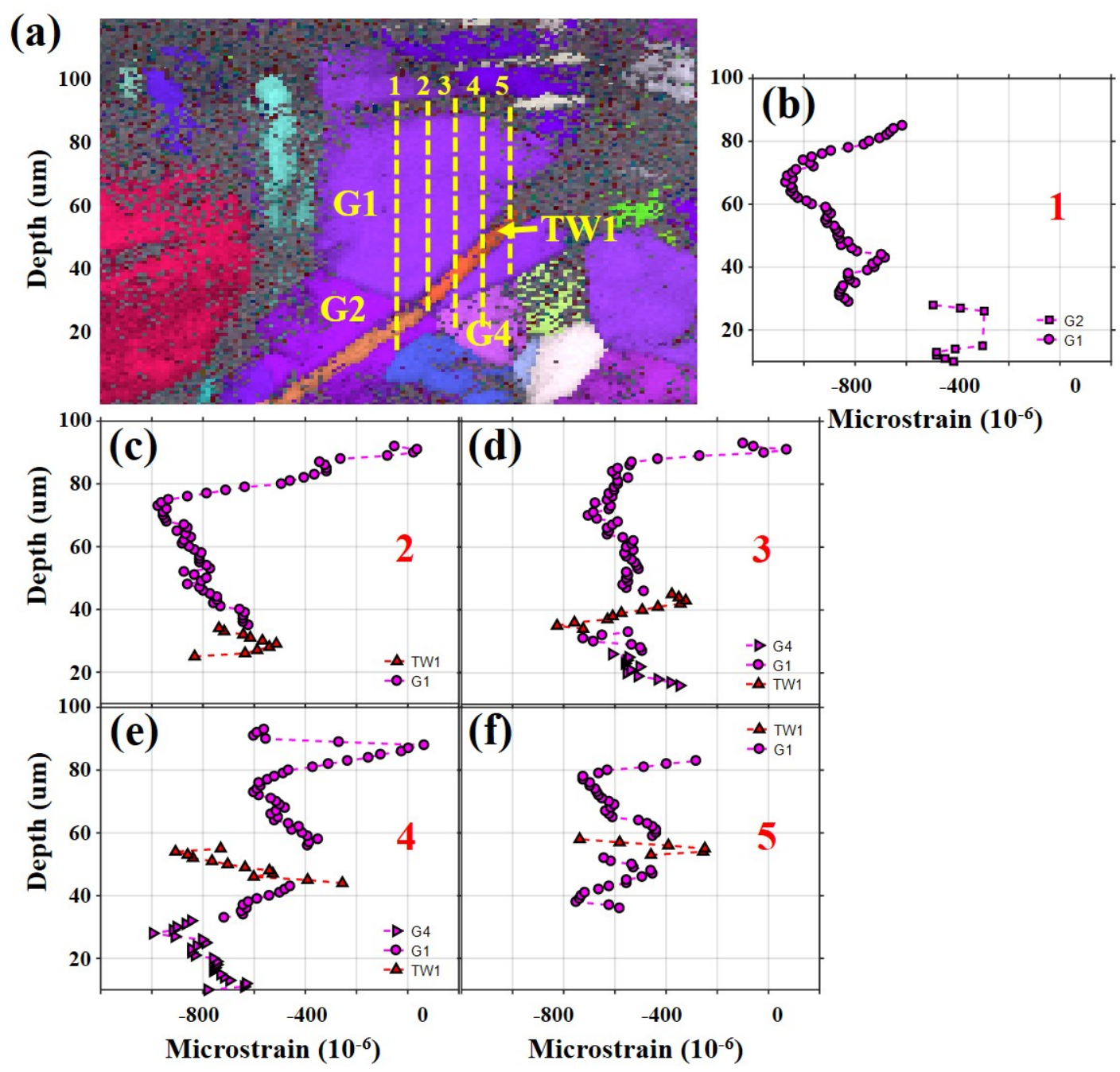

Figure 5. Lattice strain distribution in twins and the matrix grains. (a) The morphology of the grains. (b-f) The monochromatic-mode $\mu$ XRD line scanning on the plane of (a) with the scanning trace indexed by yellow dot lines.

The migration of the incoherent structure is usually accompanied with the formation of non-equilibrium basal stacking faults (SFs) in newly formed $\{10-12\}<10-11>$ extension twins, which have been confirmed by many researches $[24,25]$, while few basal SFs were observed inside the corresponding matrix grains as the TEM 
shown. Basal SFs nucleate with one end being anchored at the moving TB while the structure of the initial TB is incoherent, resulting in the micron-width SFs and presenting a morphology with straight lines well aligned to the trace of the basal planes. The non-equilibrium basal SFs inside the twin may act as obstacles hindering the subsequent movement of dislocations on the prismatic and pyramidal slip planes.

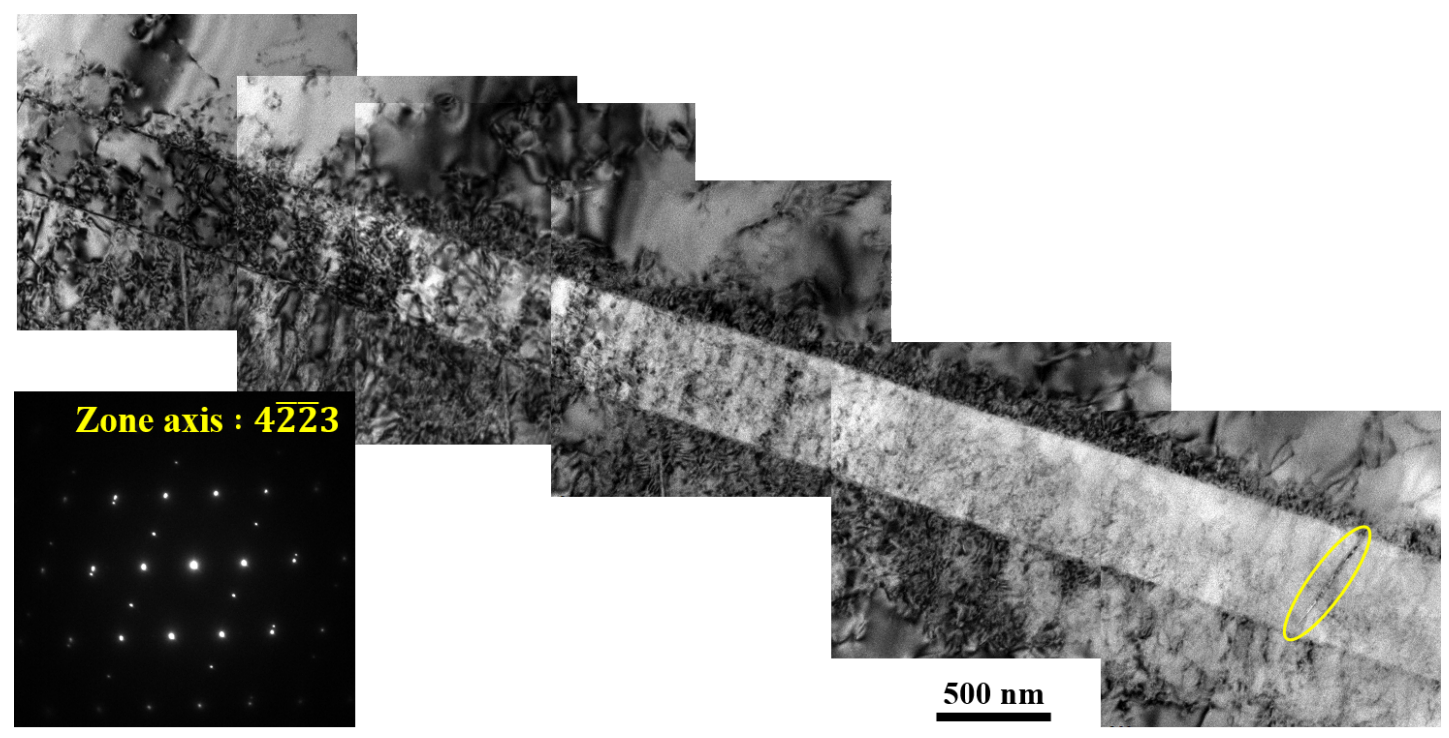

Figure 6. Twinning dislocations and SFs near TBs.

Figure 6 shows the TEM dark field image of an extension twin in the $2.4 \%$ deformed pure titanium sample. High density of dislocations observed on/near the TBs play a modulating role in balancing the local strain/stress heterogeneity of the TBs, especially in the place with incoherent structures. Some SFs crossing the twinning band were also observed and were marked by yellow circle in Figure 6. This is consistent with the above results derived from the $\mu \mathrm{XRD}$ experiments.

\section{Conclusion}

In this paper, the synchrotron based techniques, i.e. HE-XRD and $\mu$ XRD were employed to study the LSP processed component and deformation twinning in pure titanium in order to reveal the lattice strain gradients and deformation microstructures and demonstrate the accommodation mechanisms of localized strains. Benefits from the advanced characterization tools and methods developed in synchrotron radiation facility will promote the development of material science and help us understand the deformation heterogeneity. 


\section{Acknowledgements}

This work was supported by the National Key Research and Development Program of China (Grant No. 2017YFA0403804), the National Natural Science Foundation of China (Grant Nos. 51527801 and 11805009), the Fundamental Reseaerch Funds for Central Universities (Grants No. FRF-TP-18-027A1) and the AeroScience Fund (Grant No. 20145374002). R.G. L. acknowledges the financial support from the Chinese Scholarship Council (CSC). The use of the Advanced Photon Source was sup- ported by the U.S. Department of Energy, Office of Science, Office of Basic Energy Sciences, under Contract No. DE-AC02-06CH11357.

\section{References}

[1] H. Paul, A. Morawiec, J.H. Driver, E. Bouzy, Int. J. Plast. 25 (2009) 1588-1608.

[2] C.S. Montross, T. Wei, L. Ye, G. Clark, Y.W. Mai, Int. J. Fatigue 24 (2002) 1021-1036.

[3] P. Peyre, R. Fabbro, Opt. Quantum Electron. 27 (1995) 1213-1229.

[4] A.K. Gujba, M. Medraj, Laser Peening Process and Its Impact on Materials Properties in Comparison with Shot Peening and Ultrasonic Impact Peening, 2014.

[5] U. Trdan, M. Skarba, J. Grum, Mater. Charact. 97 (2014) 57-68.

[6] X.C. Zhang, Y.K. Zhang, J.Z. Lu, F.Z. Xuan, Z.D. Wang, S.T. Tu, Mater. Sci. Eng. A 527 (2010) 34113415 .

[7] K. Kasaba, T. Sano, S. Kudo, T. Shoji, K. Katagiri, T. Sato, J. Nucl. Mater. 258-263 (1998) 2059-2063.

[8] J. Ruschau, R. John, S. Thompson, T. Nicholas, Int. J. Fatigue 21 (1999) 199-209.

[9] Y.W. Fang, Y.H. Li, W.F. He, P.Y. Li, Mater. Sci. Eng. A 559 (2013) 683-692.

[10] R. Su, L. Li, Y.D. Wang, Z.H. Nie, Y. Ren, X. Zhou, J. Wang, AIP Adv. 8 (2018) 055126.

[11] R. Li, Y.D. Wang, W. Liu, C. Geng, Q. Xie, D.E. Brown, K. An, Acta Mater. 165 (2019) 336-345.

[12] R. Li, Q. Xie, Y.-D. Wang, W. Liu, M. Wang, G. Wu, X. Li, M. Zhang, Z. Lu, C. Geng, T. Zhu, Proc. Natl. Acad. Sci. 115 (2018) 483-488. 
[13] Z.W. Zhu, C.Y. Xiong, J. Wang, R.G. Li, Y. Ren, Y.D. Wang, Y. Li, Acta Mater. 154 (2018) 45-55.

[14] S. Li, T. Wang, Q. Tan, R. Li, Y. Wang, X. Wang, Y. Ren, Y. Wang, Mater. Sci. Eng. A 739 (2019) 264 271.

[15] B.C. Larson, W. Yang, G.E. Ice, J.D. Budai, J.Z. Tischler, Nature 415 (2002) 887-890.

[16] L.E. Levine, B.C. Larson, W. Yang, M.E. Kassner, J.Z. Tischler, M.A. Delos-Reyes, R.J. Fields, W. Liu, Nat. Mater. 5 (2006) 619-622.

[17] K. Schiffner, C. Droste, Comput. Struct. 72 (1999) 329-340.

[18] K. Zhu, C. Jiang, Z. Li, L. Du, Y. Zhao, Z. Chai, L. Wang, M. Chen, Mater. Des. 107 (2016) 333-340.

[19] J.W. Christian, S. Mahajan, Prog. Mater. Sci. 39 (1995) 1-157.

[20] A.G. Crookerf, Proc. R. Soc. London. Ser. A. Math. Phys. Sci. 288 (1965) 240-255.

[21] B.Y. Liu, J. Wang, B. Li, L. Lu, X.Y. Zhang, Z.W. Shan, J. Li, C.L. Jia, J. Sun, E. Ma, Nat. Commun. 5 (2014).

[22] Q. Sun, X.Y. Zhang, J. Tu, Y. Ren, H. Qin, Q. Liu, Philos. Mag. Lett. 95 (2015) 145-151.

[23] L. Balogh, S.R. Niezgoda, A.K. Kanjarla, D.W. Brown, B. Clausen, W. Liu, C.N. Tomé, Acta Mater. 61 (2013) 3612-3620.

[24] Q. Sun, X. Zhang, Y. Shu, L. Tan, Q. Liu, Mater. Lett. 185 (2016) 355-358.

[25] W. Wang, P. Jiang, F. Yuan, X. Wu, Philos. Mag. 98 (2018) 1186-1203. 